\title{
Editorial for special issue on Virtual Reality and Augmented Reality
}

\author{
Wadee Alhalabi ${ }^{1} \cdot$ Miltiadis D. Lytras $^{1}$
}

Published online: 8 August 2019

๑) Springer-Verlag London Ltd., part of Springer Nature 2019

Virtual and Augmented Reality (VR/AR) promotes a new paradigm for Human Computer Interaction and the design of Pervasive Systems. In a way, VR/AR defines a new form of sociotechnical systems, offering high impact in the design, implementation and provision of services and experiences.

The objective of the special issue is to communicate and disseminate recent computer engineering and VR/AR research and application development that demonstrate the capacity of VR/AR to change radically the user experience in the near future.

Human activities, including business, commerce, education, health and entertainment, will face tremendous changes due to emerging ubiquitous radical VR/AR systems. From solutions related to short VR experiences to fully functional mobile virtual human networks and HoloLens applications, a new era of human experience has begun. In this context of VR/AR-enabled human interactions, there are requirements for content development, new standards and innovative strategies and models for personalization, unique value proposition and innovations in business cases.

Current applications of VR/AR and VR/AR industry worldwide present a very interesting picture. Most of the dominant hardware providers provide advanced headsets and other kinesthetic wearables for VR/AR, and software houses provide sophisticated applications in a variety of interactions related to simple or sophisticated human activities. For example, marketing functions in most of the famous brands consider VR/AR as an effective marketing channel, initially converging with but then perhaps moving beyond social networks and mobile and ubiquitous technologies, offering added value to the customer experience.

Several small-/medium-scale systems already provide a range of services to different stakeholders, including merchants, customers, intermediaries and professionals. A key challenge for VR/AR to become more mainstream in this

Miltiadis D. Lytras

miltiadis.lytras@gmail.com

1 King Abdulaziz University, Jeddah, Saudi Arabia area is for traditional commerce and e-commerce to be critically enriched by technology-enabled components offering VR/AR experiences. The potential impact of VR/AR on retail/commerce is reflected in surveys that indicate that VR/ AR can change radically retail/commerce business models, suggesting the capacity of the VR/AR technology to redefine how commerce is conducted.

The purpose of the special issue is present state-of-the art approaches to, and examples of, of VR/AR systems and components for different aspects of human activities and retail/commerce.

After a rigorous peer review process, of three rounds, we are happy to share with you the final collection of accepted papers. As a minimal contribution of this special issue to the research community and audiences of Virtual Reality journal, we would like to highlight some emerging research areas for future Virtual Reality and Augmented Reality Research:

- Integration of VR/AR with sophisticated artificial intelligence and machine learning for advanced visual recommender systems for different domains of human activity (Lytras et al. 2017).

- Annotation of VR/AR content and integration to smart cities applications for enhanced citizens experience.

- Adoption of social networking research to VR/AR-integrated applications and services for the development of an evolutionary VR/AR social network for the management of the virtual self (Lytras et al. 2015a, b, 2016).

- Progressive VR/AR for retail and commerce: the VR/AR superstores for the future.

- Application of advanced visual data processing for sentiment analysis in VR/AR applications.

- Evolution of a cloud-based ecosystem of VR/AR applications for commerce, health, business, tourism and entertainment.

We want to thank the experts, academics and scholars that served the demanding, high-quality review process and the Editor in Chief. Dr. Dan Ballin for his constructive 
supervision and support in all the stages of the development of our special issue.

\section{References}

Lytras MD, Al-Halabi W, Zhang JX, Masud M, Haraty RA (2015a) Enabling technologies and business infrastructures for next generation social media: big data, cloud computing, internet of things and virtual reality. J UCS 21(11):1379-1384

Lytras MD, Mathkour HI, Abdalla HI, Al-Halabi W, Yáñez-Márquez C, Sean WMS (2015b) An emerging-social and emerging computing enabled philosophical paradigm for collaborative learning systems: toward high effective next generation learning systems for the knowledge society. Comput Hum Behav 51:557-561. https ://doi.org/10.1016/j.chb.2015.06.004

Lytras MD, Damiani E, Mathkour H (2016) Virtual reality in learning, collaboration and behaviour: content, systems, strategies, context designs. Behav IT 35(11):877-878. https://doi.org/10.1080/01449 29X.2016.1235815

Lytras M, Raghavan V, Damiani E (2017) Big data and data analytics research: from metaphors to value space for collective wisdom in human decision making and smart machines. Int J Semant Web Inf Syst 13(1):1-10. https://doi.org/10.4018/IJSWIS.2017010101

Publisher's Note Springer Nature remains neutral with regard to jurisdictional claims in published maps and institutional affiliations. 